POLLACK PERIODICA

An International Journal for Engineering and Information Sciences

DOI: $10.1556 / 606.2018 .13 .2 .2$

Vol. 13, No. 2, pp. 15-26 (2018)

www.akademiai.com

\title{
MEASURING AND SIMULATING MAGNETIC CHARACTERISTICS USING EPSTEIN FRAME
}

\author{
${ }^{1}$ Zoltan NEMETH, ${ }^{2}$ Miklos KUCZMANN \\ ${ }^{1,2}$ Department of Automation, Széchenyi István University, Egyetem tér 1 \\ H-9026 Győr, Hungary, e-mail: 'nemeth.zoltan@sze.hu, ${ }^{1}$ kuczmann@maxwell.sze.hu
}

Received 30 August 2017; accepted 16 January 2018

\begin{abstract}
The paper discusses the standard of the Epstein frame that has been used to measure magnetic characteristics of the core made of material M250-35A supplied by different frequencies between 1-400 Hz. The measuring program has been built in LabVIEW including a control, filter and data save section as well. COMSOL Multiphysics $4.3 \mathrm{~b}$ has been chosen as simulation environment, in which the Jiles-Atherton hysteresis model has been implemented.
\end{abstract}

Keywords: Epstein frame, Magnetic characteristics, Jiles-Atherton model, COMSOL Multiphysics

\section{Introduction}

The objective of this work was to build up an Epstein frame, which has been used to measure magnetic characteristics of different type of core supplied by current on the frequency range of $1 \mathrm{~Hz}-400 \mathrm{~Hz}$. The frame consists of 4 primary coils with 250 turns each. The power generator is connected to the primary winding supplied by sinusoidal current generator. The device has 4 secondary coils as well with 300 turns of each, and another connection points at 3,15,150 turns. Fig. 1 shows the scheme of the completed device.

To use the frame properly, a measuring program in LabVIEW has been implemented. Various parameters have been obtained by the program, for example the magnetic field and magnetic flux density to display the hysteresis characteristics. It also has a control mechanism and a data log option after the measurements. 


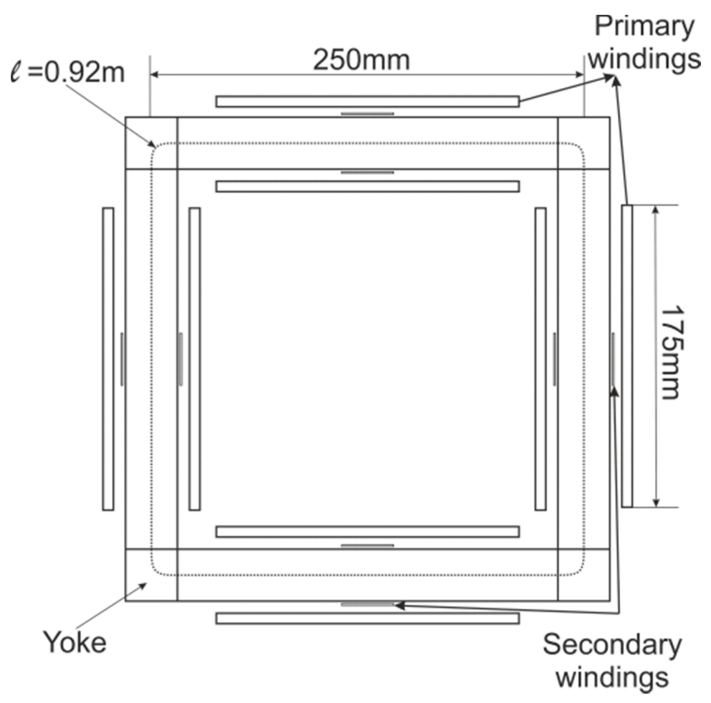

Fig. 1. The scheme of the Epstein frame [1]

The two most well-known hysteresis identification methods are the Preisach model and the Jiles-Atherton model. For this work, the Jiles-Atherton model had been chosen [2]. The advantage of this model is that the implementation of Jiles-Atherton model into COMSOL Multiphysics $4.3 \mathrm{~b}$ is easier. The differential equation can be implemented as a Partial Differential Equation (PDE) and the parameter identification equations had been defined as variables. From the measurement results the identification of the Jiles parameters $\left(a, \alpha, c, k, M_{s}\right)$ could be performed. After the Jiles-Atherton model implementation into COMSOL, the simulation with the same excitation and geometry could be realized.

\section{The Epstein frame}

The Epstein frame is used to measure magnetic properties of electrical steels and strips. The standard says, the steels weight has to be $1 \mathrm{~kg}$, the excitation has to be sinusoidal voltage, and some other data are already shown in Fig. 1.

At the beginning the coil parameters had to be calculated. The overall primary coils have 1000 turns and the secondary coils have 1200. With these parameters the measurements between $1-400 \mathrm{~Hz}$ can be performed and the magnetic field can be up to $10.000 \mathrm{~A} / \mathrm{m}$ while the magnetic flux density can reach about $2 \mathrm{~T}$ [3], [4].

Unfortunately, in the laboratory only current generator is available, which means that control section into LabVIEW program had to be implemented to obtain sinusoidal induced voltage. There can be some difference in the results, because the measurements were made with only 36 iron sheets with $900 \mathrm{~g}$ weight.

In Fig. 2 the completed frame can be seen. 


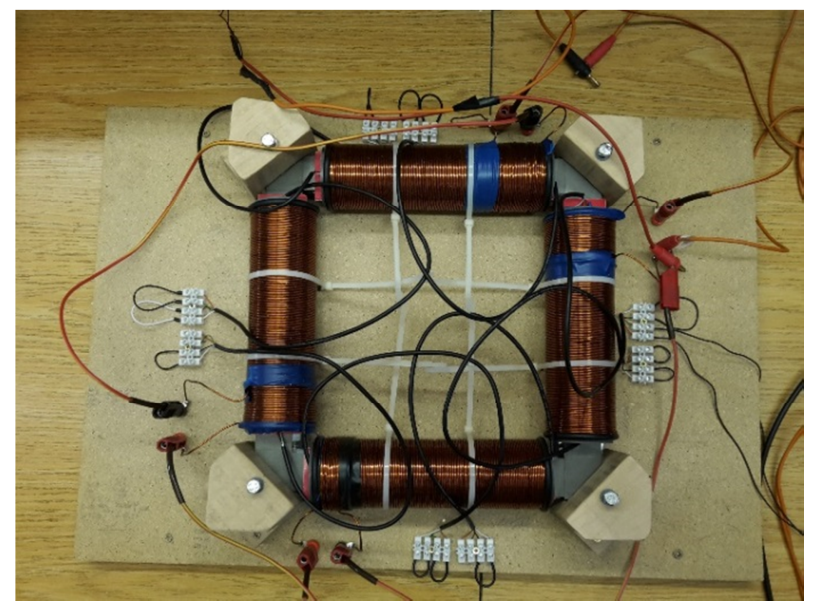

Fig. 2. The measurement ready Epstein frame

\section{The measurement program in LabVIEW}

LabVIEW has a graphical programming interface, where Virtual Instruments (VIs) for any kind of work can be realized. Suitable hardware is needed to can make measurements, which is represented by the connection between Epstein frame and VI [5], [6].

The program has been built up by 7 important sections as it is shown in Fig. 3 .

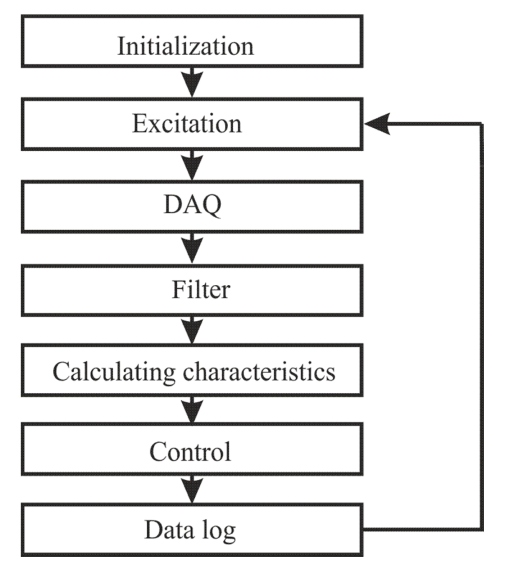

Fig. 3. The structure of the measuring program

In the initialization section the physical channels can be setup, variables and plots are cleared. As it was mentioned in session 2, the excitation current is sinusoidal between $1 \mathrm{~Hz}$ and $400 \mathrm{~Hz}$ frequency. The Data Acquisition (DAQ) section contains the output and input signal processing. One of the most important things in low frequency 
measurement is to remove the noise from the measured data. For this process a Fourier series expansion of the measured signals has been implemented. The difference between the measured signal before and after the filtering is shown in Fig. 4 and Fig. 5.

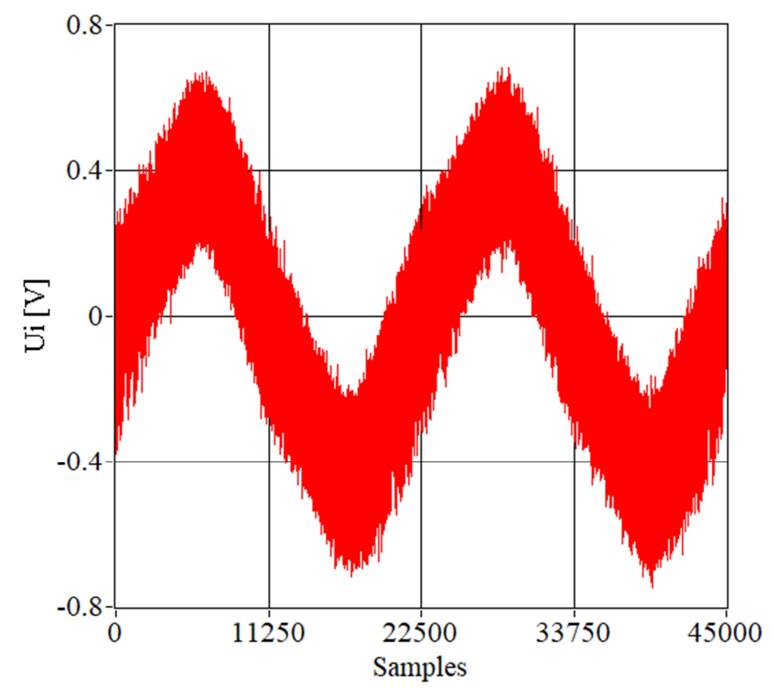

Fig. 4. Induced voltage without any filter

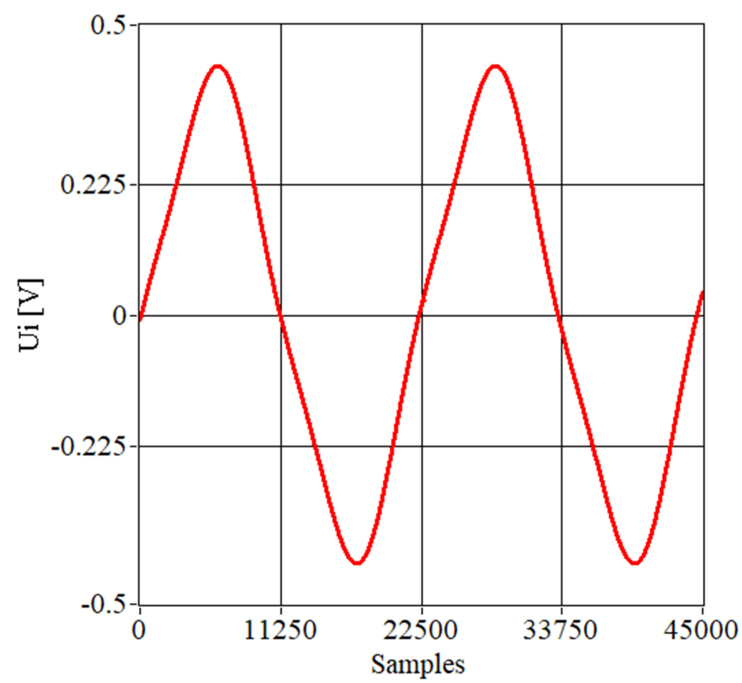

Fig. 5. Induced voltage after filtering

After filtering, the program calculates the magnetic field from the excitation and the magnetic flux density from the induced voltage of the secondary coil. The control section takes care of the sinusoidal waveform of the magnetic flux density. After the 
measurements, every single waveform and parameter with timestamp can be saved into a text file. Later on while making documentation, the necessary data have to be imported into MATLAB or LabVIEW to analyze and compare the results with different amplitudes and frequency. In Fig. 6 the graphical user interface can be seen while some measurements are performed at frequency of $5 \mathrm{~Hz}$.

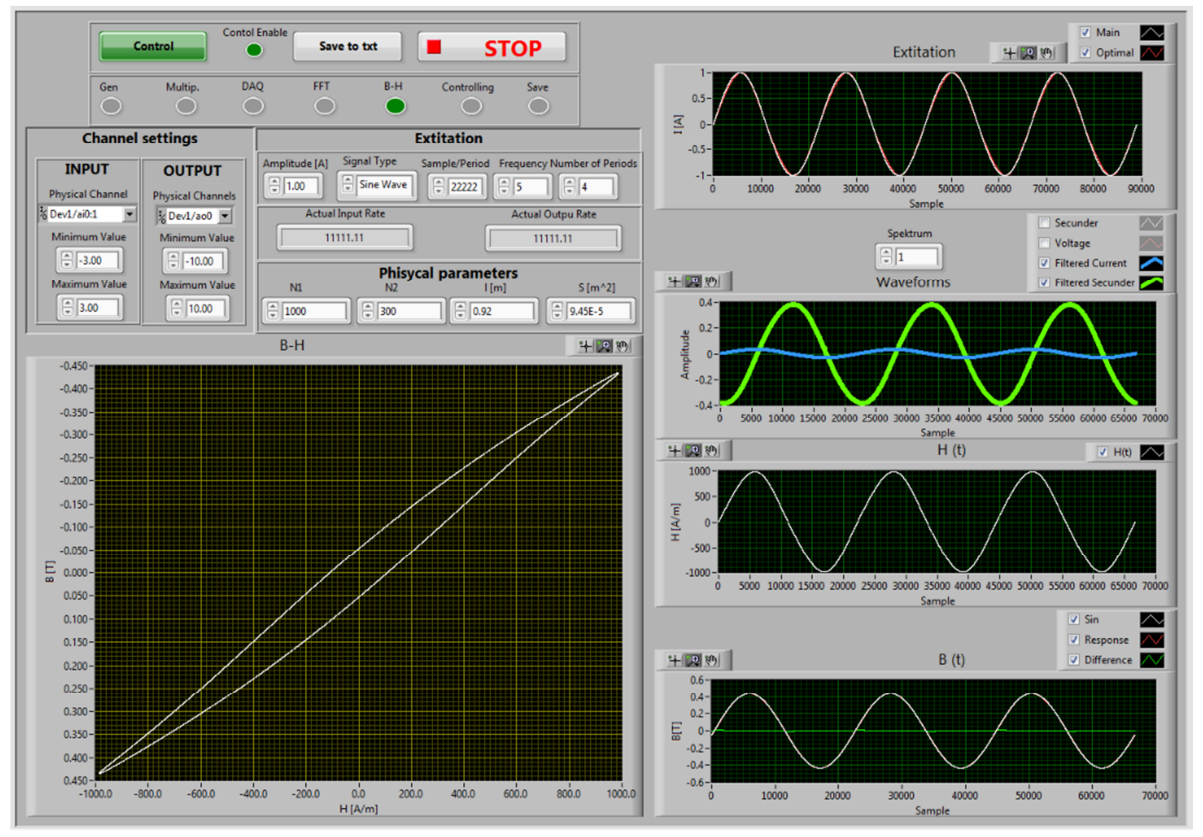

Fig. 6. The graphical user interface while measuring

\section{The Jiles-Atherton hysteresis model}

While measuring ferromagnetic materials hysteresis phenomenon can be observed. The relation between the magnetic flux density $B$ and magnetic field intensity $H$ the following relation is represented [7], [8]:

$$
B=\mu_{0}(H+M),
$$

where $M$ is the magnetization of the material, and $\mu_{0}$ is the permeability of vacuum. The Langevin model for paramagnets, if there is no hysteresis loss, can be formulated as

$$
M_{a n}=M_{S}\left[\operatorname{coth}\left(\frac{H}{a}\right)-\frac{a}{H}\right] \text {. }
$$


Here $M_{S}$ is the saturation magnetization, and $a$ is a model parameter. Because in ferromagnetic materials the moments next to each other have an interaction, the Weiss correction in (2) is usually applied. If $(H+\alpha M)$ is small enough (2) is modified to

$$
M_{a n}=M_{S} \frac{H+\alpha M}{3 a} .
$$

where $\alpha$ is another model parameter.

The Jiles-Atherton model says that the magnetization is represented as the sum of a reversible and an irreversible component, i.e.

$$
M=M_{\text {rev }}+M_{\text {irr }},
$$

where the reversible magnetization can be calculated as

$$
M_{\text {rev }}=c\left(M_{\text {an }}-M_{\text {irr }}\right) .
$$

Here $c$ is the reversible coefficient. The magnetization can be expressed from (4) and (5) as

$$
M=c M_{a n}+(1-c) M_{i r r} .
$$

From the energy balance in the material the following relation can be formulated

$$
\mu_{0} \int M_{i r r}(H) d H_{e}=\mu_{0} \int M_{a n}(H) d H_{e}-\mu_{0} \int k \delta \frac{d M_{i r r}}{d H_{e}} d H_{e}
$$

which leads consequently to

$$
M_{a n}(H)=M_{i r r}(H)+k \delta \frac{d M_{i r r}}{d H_{e}}
$$

where $k$ is a model parameter; $\delta$ is a signum function of the derivative magnetic field and $H_{e}=H+\alpha M$ is the effective field. At last, the differential equation of the JilesAtherton model can be expressed from (6) and (8) after a derivation as

$$
\frac{d M}{d H}=\frac{c \frac{d M_{a n}}{d H_{e}}+(1-c) \frac{d M_{i r r}}{d H_{e}}}{1-a c \frac{d M_{a n}}{d H_{e}}-\alpha(1-c) \frac{d M_{i r r}}{d H_{e}}}
$$


The mentioned parameters can be defined from the experimental data. To obtain the values, 5 equations must be taken into iteration [9]:

$$
\begin{aligned}
& c=\frac{3 a \chi_{i n}}{M_{S}}, \\
& a=\frac{M_{S}}{3}\left(\frac{1}{\chi_{a n}}+\alpha\right), \\
& k=\frac{M_{a n}\left(H_{c}\right)}{\chi_{H_{c}}-c \frac{d M_{a n}\left(H_{c}\right)}{d H} \frac{1+\alpha}{1-c},} \\
& \alpha=\frac{k(1-c)}{M_{r}-M_{a n}}-\frac{\chi_{M_{r}}-c \frac{d M_{a n}}{d H}}{1-c} \\
& a=\frac{M_{S}}{3 \chi_{i n}}\left[1-\frac{M_{a n}\left(H_{m}\right)-M_{m}}{k \chi_{m}}\left(\alpha \chi_{m}+1\right)\right] .
\end{aligned}
$$

where $\chi_{i n}$ is the initial normal susceptibility; $\chi_{a n}$ is the initial anhysteretic susceptibility; $\chi_{H_{c}}$ is the differential susceptibility at the coercive point; $\chi_{M_{r}}$ is the differential susceptibility at remanence; $\chi_{m}$ is the maximum differential susceptibility; $M_{r}$ is the remanence magnetization; $H_{c}$ is the coercivity; $M_{m}$ is the maximum magnetization and $H_{m}$ is the maximum magnetic field.

The scheme of the used iteration is shown in the Fig. 7 [10], [11].

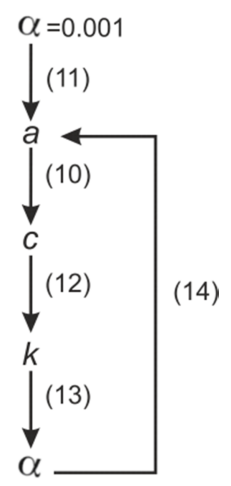

Fig. 7. Iteration for determining model parameters 
As it can be seen, an initial value for $\alpha=0.001$ has been applied. Using equations (11), (10), (12) and (13) a new value for $\alpha$ has been obtained. Using (14) and repeating this calculation until the parameters change. After the iteration completed, the JilesAtherton parameters can be obtained as it is shown in Table I.

Table I

Values of the model parameters

\begin{tabular}{|c|c|}
\hline Parameter & Value \\
\hline$a$ & $1425 \mathrm{~A} / \mathrm{m}$ \\
$\alpha$ & 0.0005 \\
$c$ & 0.1 \\
$k$ & $255 \mathrm{~A} / \mathrm{m}$ \\
$M_{s}$ & $1500000 \mathrm{~A} / \mathrm{m}$ \\
\hline
\end{tabular}

These parameters are constant except $k$, because it depends on the frequency, $k=255 \mathrm{~A} / \mathrm{m}$ is used with $1 \mathrm{~Hz}$ excitation.

\section{Simulation of the Epstein frame by FEM}

Since the distribution of the magnetic field in the Epstein frame is near homogeneous, so the correctness of the hysteresis simulation in computation and measurements are going to be performed. The COMSOL Multiphysics $4.3 \mathrm{~b}$ is numerical based software for modeling and simulating any kind of physics [12]. For simulation the Epstein frame has been modeled in 2D. The geometry was drawn in COMSOL using the build in modules. Meshing the geometry is very important because of the resolution and the precision of the surface plots and simulation time. The optimized mesh for the simulation is shown in Fig. 8. As it can be seen, the element size of the core has been set to extremely fine to get exact results at the edges as well. The complete mesh consists of 17038 domain elements and 1468 boundary elements. The number of degrees of freedom solved for 279554. The simulation time took 2049 seconds using Intel Core $17-4720 \mathrm{HQ}$ processor, which has 4 cores with a maximum of 3.6 GHz frequency. The implementation of the Jiles-Atherton model into COMSOL will not be described in this paper; it has been shown in detail in [13], [14], [15].

\section{Results}

The measurement has been performed using material M250-35A produced by ArceroMittal. Measurements and simulations have been performed applying 7 different frequencies with 8 different amplitudes. It is too much to represent all of them, so only the result at frequency of $1 \mathrm{~Hz}$ is shown. During measurements sinusoidal current excitation has been applied with a control mechanism to obtain sinusoidal induced voltage. Before saving the result the measurement program had been run for 15 periods long. For measuring the minor curves 8 different measurement have been applied with different amplitudes, starting with $0.52 \mathrm{~A}$ and exponentially growing until $8.5 \mathrm{~A}$ to 
obtain monotonously increasing magnetic flux density from $0.2 \mathrm{~T}$ up to $1.6 \mathrm{~T}$. From the induced voltage signal the first period had been removed before Fast Fourier Transform (FFT) because of the transient processes, where every harmonic component has been removed. The result is shown in Fig. 9.

Comparison of measured and simulated results is shown in Fig. 10a and Fig. $10 \mathrm{~b}$.

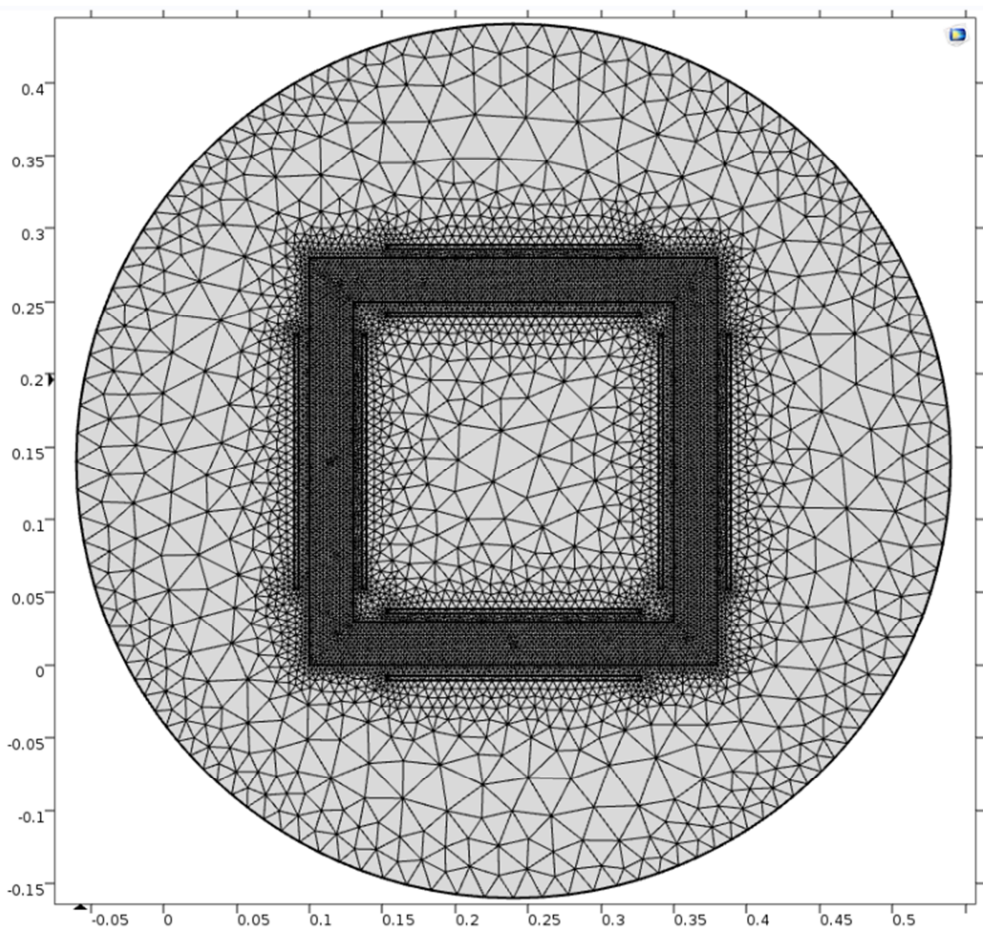

Fig. 8. Meshed geometry of the Epstein frame

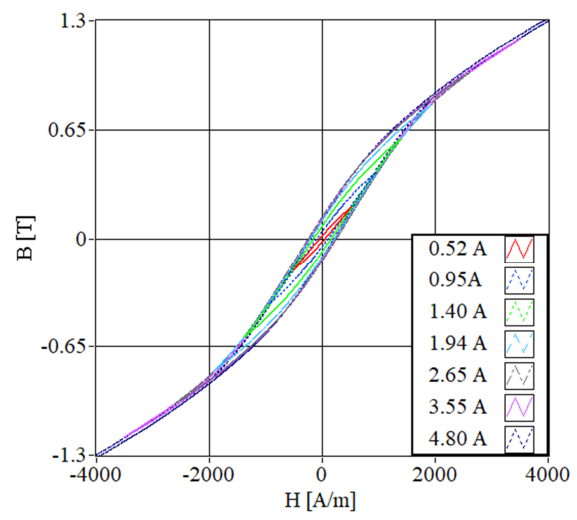

Fig. 9. Measurement result in $1 \mathrm{~Hz}$ frequency 


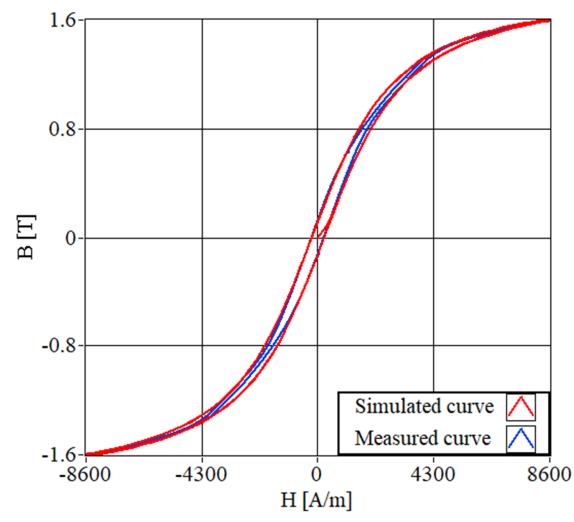

a)

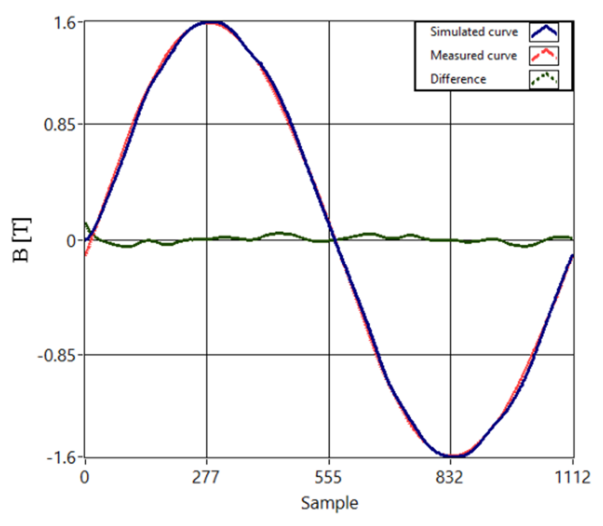

b)

Fig. 10. Comparison of measured and simulated results

As it can be seen, there is a difference between the two curves, especially in the case of the higher amplitudes, where the measured curve is completely narrowed. As it can be seen in Fig. 10b, there is minimal difference when the magnetic induction is close to zero or its maximum, at the other parts of the curve, there are some difference with a maximum of $0.049 \mathrm{~T}$. This difference can be caused, maybe, by the control mechanism.

Fig. 11 shows the absolute value of the magnetic flux density at $t=0.75 \mathrm{~s}$.

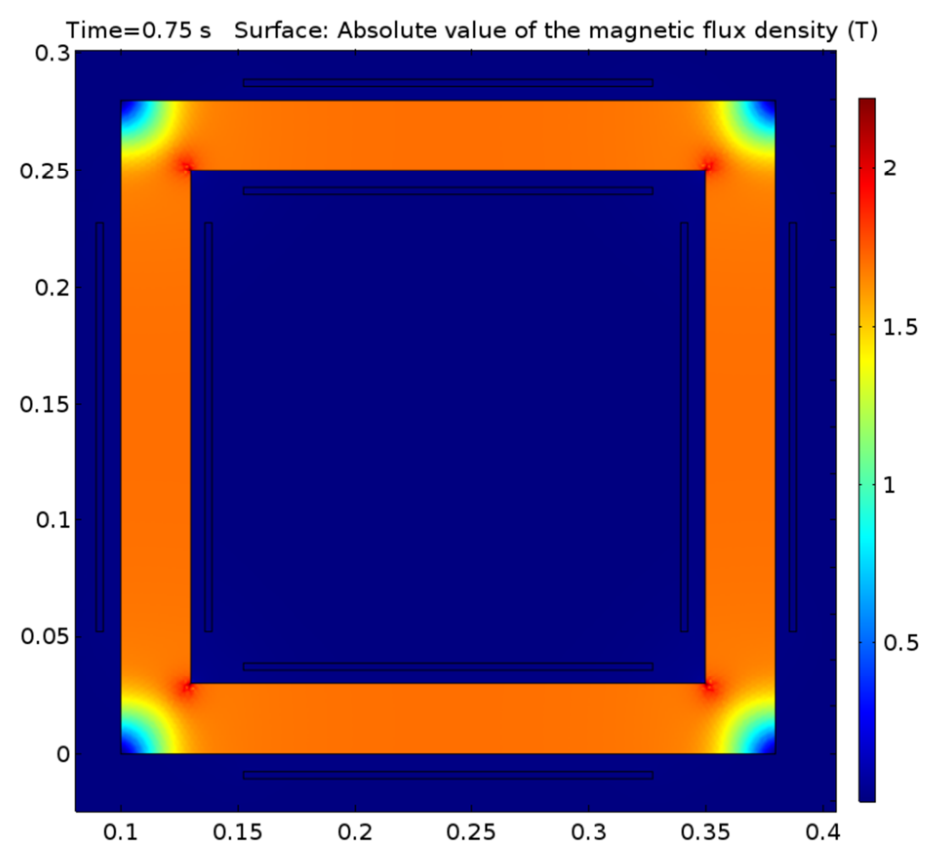

Fig. 11. Simulated absolute value of the magnetic flux density at $t=0.75 \mathrm{~s}$ 
The simulation result shows that at the outer edges of the frame the magnetic induction is minimal, less than $0.2 \mathrm{~T}$ and increasingly growing toward the inner corners up to $2.06 \mathrm{~T}$. At the other parts of the iron the magnetic flux density is evenly distributed. Using FEM for electromagnetic simulations is highly recommended after measurements to can confirm that the result is valid.

\section{Conclusion}

The objective of this work is to introduce the basic knowledge of the Epstein frame, and to present the own made device, how to build a measuring program for electromagnetic measurements. The second part of the paper presented the necessary equations of the Jiles-Atherton model. The simulation environment is COMSOL Multiphysics $4.3 \mathrm{~b}$, where the hysteresis model has been built in, but the implementation of the model is not presented. At last the measurement result at $1 \mathrm{~Hz}$ has been compared with the simulations.

In the future, the Epstein frame will be useful at measuring different type of electromagnetic steels and comparing them to each other. The advantage of using COMSOL Multiphysics $4.3 \mathrm{~b}$ for simulation is that the implementation of the JilesAtherton model is not complicated; moreover, the simulation gives accurate result. The only disadvantage of the model is the definition parameters are sometimes cumbersome. The vector extension of the hysteresis measurement will also be in the focal point of research.

\section{Acknowledgement}

This work has been supported by the ÚNKP-17-4 New National Excellence Program of the Ministry of Human Capacities.

\section{References}

[1] IEC 60404-2 Magnetic materials, Part 2, Methods of measurement of the magnetic properties of electrical steel sheet and strip by means of an Epstein frame, International Electrotechnical Comission Std, 1996.

[2] Fallahnejad M., Afrakhte H. The comparison of two approaches Jiles-Atherton and Preisach in simulating hysteresis cycle, 2nd International Conference on Knowledge-Based Engineering and Innovation (KBEI), Teheran, Iran, 5-6 November 2015, pp. 710-713.

[3] Bastos J. P. A., Sadowski N. Electromagnetic modeling by finite element methods, Marcel Dekker Inc, New York, 2003.

[4] Fodor Gy. Electromagnetic fields (in Hungarian), Mủegyetemi Kiadó, Budapest, 1996.

[5] Polik Z., Kuczmann M. Measurement and control of scalar hysteresis characteristics, Pollack Periodica, Vol. 2, No. 2, 2007, pp. 27-37.

[6] Tang Q., Anderson P., Wang Z., Moses A., Jarman P. Measurement of magnetic properties of electrical steels at high flux densities using improved single sheet tester, 18th International Symposium on High Voltage Engineering, Seoul, Korea, 25-30 January 2013, pp. 27-32. 
[7] Jiles D., Atherton D. Ferromagnetic hysteresis, IEEE Trans. Magn, Vol. 19, No. 5, 1983, pp. 2183-2185.

[8] Ivanyi A. Hysteresis models in electromagnetic computation, Akademiai Kiado, Budapest, 1997.

[9] Kis P. Jiles-Atherton model implementation to edge finite element method, PhD Thesis, Budapest University of Technology and Economics, 2007.

[10] Nicusor P, Ovidiu C. Algorithm to describe magnetization curves by using Jiles Atherton model, Pollack Periodica, Vol. 5, No. 3, 2010, pp. 155-165.

[11] Friedl G., Kuczmann M. Identicication of Scalar Jiles-Atherton hysteresis model parameters with a new particle behavior based metaheuristic algorithm, Przeglad Elekttrotechniczny, Vol. 12, 2015, pp. 87-90.

[12] https://www.comsol.com/comsol-multiphysics/ (last visited 25 August 2017).

[13] COMSOL Multiphysics, Modeling hysteresis effects, Solved with COMSOL multiphysics 3.5a, 2008.

[14] Kovacs G, Up-to-date finite element based simulation for permanent magnet, Acta Technica Jaurisnensis, Vol. 7, No. 2, 2014, pp. 172-182.

[15] M. Holters, U. Zölzer, Circuit simulation with inductors and transformers based on the Jiles-Atherton model of magnetization, 19th International Conference on Digital Audio Effects (DAFx-16), Brno, Czech Republic, September 5-9, 2016. 